\title{
Crafting On-Skin Interfaces: An Embodied Prototyping Journey
}

Juan Restrepo-Villamizar

Eindhoven Universitiy of Technology

Eindhoven, The Netherlands

j.c.restrepo.villamizar@tue.nl

\author{
Steven Vos \\ Eindhoven Universtiy of Technology \\ Eindhoven, The Netherlands \\ s.vos@tue.nl

\section{Evert Verhagen} \\ Amsterdam University Medical Center \\ Amsterdam, The Netherlands \\ e.verhagen@amsterdamumc.nl
}

Carine Lallemand

Eindhoven Universtiy of Technology

Eindhoven, The Netherlands

c.e.lallemand@tue.nl

\section{ABSTRACT}

This pictorial presents a design exploration of On-Skin Interfaces for recreational running. By integrating principles of interaction design, art and psychology, we explore the design of unconventional interfaces that facilitate the intuitive understanding of biofeedback and physiological-related information. We explored how principles from agency and bodily ownership can be applied in the design of sportrelated wearables. Through our embodied prototyping journey, we gained insights on the implications of using the skin as an interactive design material. We focused on diverse materiality explorations to uncover and highlight the possibilities and challenges of materializing both functional and appealing On-Skin Interfaces. We synthesize and reflect on our theoretical and practical explorations and deliver actionable insights for this growing field of bodily and unconventional interfaces.

\section{Authors Keywords}

Wearable computing; On-skin Interfaces; Embodied Interaction; Fabrication; Recreational Running.

\section{CSS Concepts}

- Human-centered computing Human computer interaction (HCI)

Permission to make digital or hard copies of part or all of this work for personal or classroom use is granted without fee provided that copies are not made or distributed for profit or commercial advantage and that copies bear this notice and the full citation on the first page. Copyrights for third-party compone DIS '21, June 28-July 2, 2021, Virtual Event, USA (C) 2021 Copyright is held

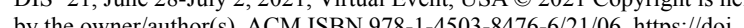
org/10.1145/3461778.3462055
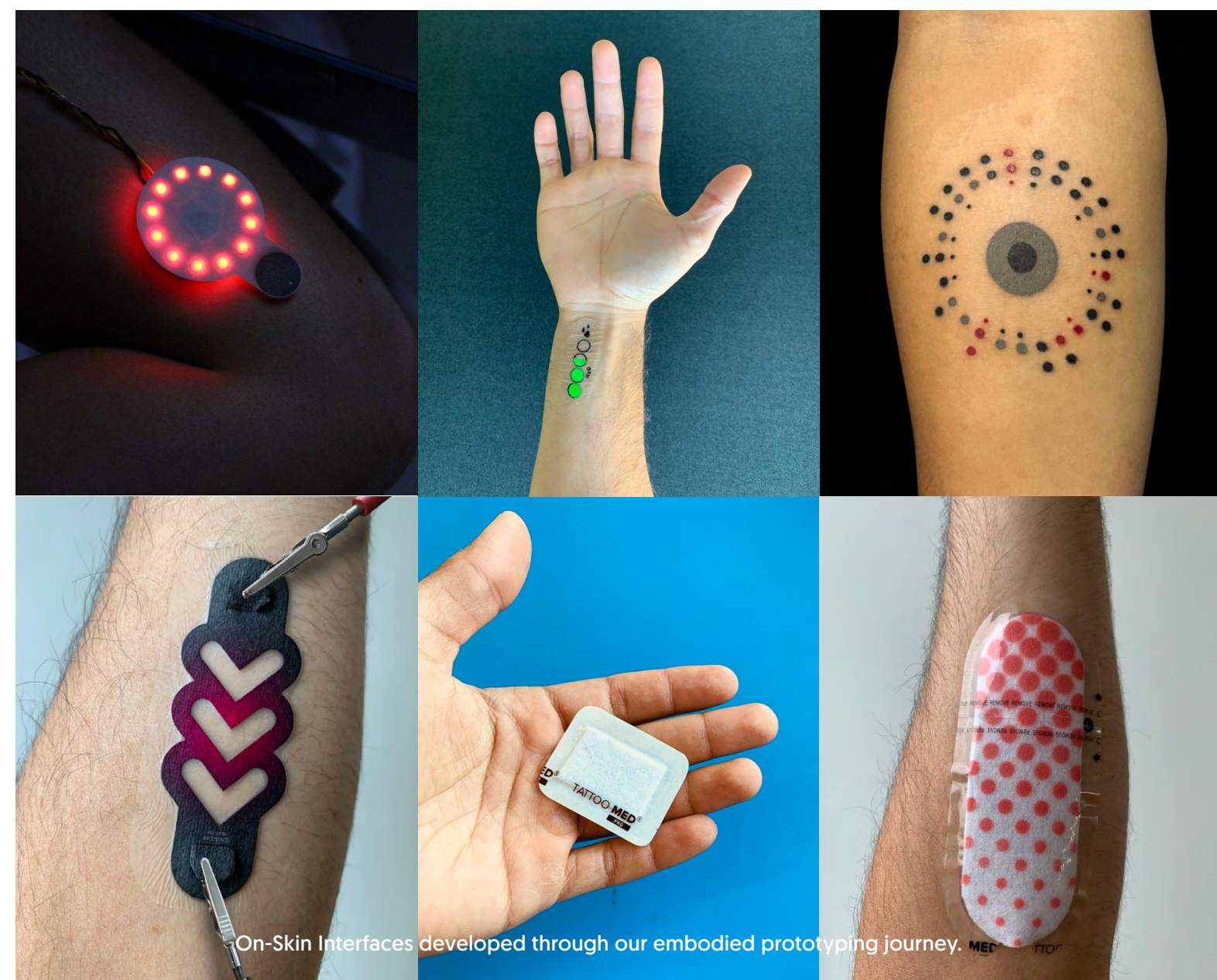


\section{ON-SKIN INTERFACES: THE HUMAN SKIN AS AN INTERACTIVE SUBSTRATE}

As the outermost layer of our body, the human skin offers possibilities to explore unconventional and novel interactive systems and modalities [16, 20, 42]. Due to the skin's intrinsic properties and the advances of the wearable technology, on-skin interfaces (OSI) have gained attention in the field of HCI [20]. With the aim of making the human body "smarter" and augmented $[20,33,42]$, OSI endow new abilities and features into the human body and enable it to overcome its organic limitations [33]. Hence, unlike add-on-and-off devices, OSI offer the possibility to be worn as a body part, bodily embedded system or prosthetic device. Despite the increasing attention from the design and HCI community to this research topic, there are diverse challenges that limit the development and implementation of OSI in real-life contexts. While several projects have focused on the development of skin-like interfaces, it is still very difficult to develop OSI systems that are robust enough to be deployed and scaled up to mass production and the consumer market.

Wearable layer DESIGNING OSI

Sportwatches, fitness

trackers, clothing

OSI

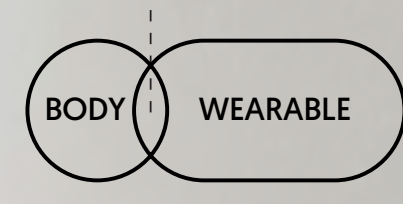

Internal layer

Tattooing

Based on the multilayer clasification of devices on the skin by lan Person
Using the human skin as a mean of interaction differs significantly from more conventional interfaces $[20,33$, $34,42]$, not only in terms of its geometry but also in its primary function and possible interaction modalities [33]. As the boundaries between the human body and technology fade and overlap, the way people interact with wearable computing technology, with themselves and with the environment reshapes [34]. As OSI reshape the boundaries of the body, they call for novel Interaction Design practices [20, 31, 33 41, 42]. Thus, some of the existing design principles for user interfaces must be questioned and adjusted before being applied to the design of OSI, as these do not account for OSI's unique physical and psychological characteristics [20, $31,33]$. Due to the integration of OSI into the human body, one interesting direction to explore their intrinsic advantages and differences over other type of interfaces, is to address their potential association with a higher bodily self-consciousness. Such association might be possible by exploring the OSI's capability of eliciting the feeling of body ownership through on-skin feedback as an illusory body continuity mechanism [7]. Body ownership refers to the feeling that the body "is mine" $[16,23]$. Human bodily experience is characterized by the immediate and continuous feeling that the body, its parts, and its sensations belong to oneself $[2,8,22$, 39]. Body continuity refers to the perception of bodily integrity. For instance, when we look to our hands, we rarely doubt whether they are part of our body or not, and therefore, we do not question the continuity and sense of ownership of our bodies. This could transform the way users interact with computing devices, and more interestingly, how people interact with themselves [2] Even though these framing is grounded on psychological principles, the design and execution of OSI can influence and be directly influenced by this theoretical approach. The materiality, appearance and aesthetic characteristic of OSI might be relevant factors to develop illusory body continuity mechanisms that blend within one's body and senses. From a design-oriented perspective, the principle of continuity in Gestalt theory [39], and the potential of creating a more humanized technological future for more engaging and bodily experiences proposed by Muller et, al. [25] intersect and support the design of OSI.

\section{OSI FOR RECREATIONAL RUNNING}

Although this pictorial compiles our material and explorative design process of OSI, our journey is framed within the context of wearables for recreational running. Recreational runners make extensive use of wearable devices such as sport watches, fitness trackers, or specialized on-body sensors to monitor their performance and health. Most of these wearable systems integrate conventional interfaces, such as (touch) screens, knobs and buttons. Hence, the user interaction occurs as an out-of-the-body-process. The interface itself, as an external source of information, defines the boundaries between the user's body and the device. It represents in 
Crafting On-Skin Interfaces:

An Embodied Prototyping Journey

[Process Overview]

an extrinsic fashion the relationship between the actions performed by the user and the information delivered by the system and relies on the cognitive skills of the users for its interpretation [7, 28]. This is the way we interact with most existing computing devices, such as smartphones, tablets, or wearables. In contrast, with OSI, user interactions occur as an intrinsic bodily process, overlapping the human body with wearable technology. The embodiment of such interfaces allows the users to use their bodies as augmented and interactive computing devices, relying on their perceptual and bodily senses. Removable tattoos to control computing devices $[18,34$, 40], electric shock (EMS) as feedback mechanisms [10, $25,40]$ or biosensors injected into the skin to detect inner body composition [1] are some examples of OSI. By translating these insights into wearables for recreational running and sports, we envisioned the potential of offering different and perhaps better ways to represent and interact with bodily-related data and feedback. As OSI transform the skin into an interactive substrate and are intended to blend seamlessly with the body, they might deliver more intuitive and meaningful feedback to support recreational athletes to better understand their body and physiological data. We conducted a researchthrough-design process to investigate these aspects. In this pictorial, we report on our explorative process to design OSI for sports applications.

\section{Tangible and Bodily Prototypes}

Due to OSI's nature, the design process of such artefacts requires a tangible and embodied approach that goes "beyond screens and is, embodied, situated and connected" [35]. To investigate such topics, researchers have explored the value of the first-person perspective $[4,11,26]$, and proposed practices and frameworks for observing and documenting these experiences in ourselves and others [4, 21, 24, 38]. Previous work also introduces methods and techniques for creating bodily designs $[4,15,36]$ and provided examples of designing and crafting wearable technologies $[14,15,4,12,14,15$,
30]. Yet, the prototyping phase is commonly discussed from a higher-level perspective or not mentioned as a core element of the design research. In this pictorial, we build on the work of Tomico et al. (2016) [35], who call for a "[migration of] the design process from the technological oriented board to a full immersion into the design context" [35] and on the design and crafting of design research artifacts that reflect on the crafting of unconventional, critical and bodily technologies $[19,27$, 32]. We invite the readers to walk through an in-depth visual report of our prototyping process for On-Skin Interfaces. We describe examples of techniques we used and reflect on the insights obtained during a rich set of experiences exploring the realm of On-Skin Interfaces, their materiality, interaction and feedback modalities, usability and prospective applications within the context of recreational running and sports. This approach enabled us to test and iterate concepts of OSI meant to be worn while running. We generated insights on how to design, implement and further develop On-Skin Interfaces, while addressing these two initial questions: (1) how to implement bodily-specific features to design On-Skin Interfaces, and (2) how, in prospective use scenarios, On-Skin Interfaces would influence the sense of bodily data ownership.

\section{OUR DESIGN JOURNEY: AN ON-SKIN IMMERSION}

Throughout the crafting process, we kept track of our prototyping stages and insights through extensive photo and video documentation and reflexive writing of blog articles and progress presentations. We synthesized this data around main topics. Therefore, the methods and exploration stages presented are not perfectly chronological or a one-to-one recounting yet act as exemplars of techniques used.

\section{First-Person Perspective}

an Embodied Point

of View Approach

\section{Empathizing with On-Skin practices}

Obtaining insights on tattooing

rituals and on-skin interventions

\section{Contextualizing}

Envisioning On-Skin Interfaces in the context of recreational running

\section{Design Principles}

Framing our research-through-design

exploration based on initial insights

\section{Ideation}

Sketching to translate design principles into design concepts through

\section{Materiality Exploration}

Prototyping OSI design concepts

OSI for Runners

Preliminar OSI design deployed in the field
Reflections

Learnings, contribution and future of OSI 


\section{FIRST-PERSON EXPERIENCES}

In early stages of the research process, we conducted several bodily experiments and crafted prototypes to investigate the complex topic of OSI through an immersive hands-on and embodied approach. Our goal was to explore different aspects linked to OSI and wearables for recreational running from a first-person perspective, enabling us to gain knowledge and insights to foreground our design process.

Visualizing Self-Related Data on the Skin. To approach the design process from an embodied point of view, we crafted stickers made of a biocompatible adhesive membrane. These stickers were designed to track differen daily activities, e.g., daily water intake or weekly training sessions (green dots marked executed training sessions or consumed water). The idea was to explore ways of visualizing "dynamic" personal data on the body. As a first-person perspective exercise, we gained insights on the social and personal implications of displaying data on the skin. People were intrigued by the purpose of the stickers, recurringly asking about their meaning. We related this to the following factors: (1) the stickers were located on visible, uncovered and meaningful body spots such as the forearms and wrists [3], (2) unlike traditional tattoos, these had extremely vivid colors, creating a high contrast with the skin tone, (3) the graphics changed over time, introducing new shapes, patterns and colors, and (4) the graphics did not represent data in a literal fash ion. Making public and constantly visible (by oneself and others) self-related data such as training habits and water intake, increased the awareness on those topics.

Tattooing, an Empathizing Exercise. Following our interest to learn more about the nature of the skin as a physical material and the tattooing as a practice to transform it, we conducted a first-person exploration with a tattoo artist and one of his clients. Inspired by the work of Eschler et al. [6] on the psychological impact of cancer survivor tattoos, we aimed to learn about the semiotics within the tattoo practice, the ritual that frames it, and the implications of displaying sensitive and private data on the skin as a public interface. We conducted a co-creation session involving a tattoo artist and a clien volunteer, who together with us designed and executed a tattoo on the skin of the client. Following the insight of Escher et, al. (2018), the tattoo design was meant to represent a meaningful life experience for the tattooed. The volunteer was asked to write a short story about an important moment of his life. He could then decide to get the text literally written on the skin, or an abstract representation of it. The participant chose the latter, so the text would not be understood by others. Being part of its design and realization process, we better grasped the intimacy a tattoo represents for both the tattoo artist and the tattooed. It also gave us the actual feeling of permanently modifying someone's body, triggering different feelings: the empathy to see the volunteer in pain and the stress realizing that there is no room for mistakes outlined the delicacy involved into a body transformation process. Furthermore, a tattoo embodies and physicalizes emotional and meaningful experiences in the form of a code understandable only by the tattoo artist and the tattooed and open for external interpretation. Through this immersion, we learned that the skin is a very delicate, valuable and sensible material that must be treated with care and precaution. Placing elements on the skin goes beyond wearability factors, and it entails a very intimate perception and interaction with this element. People might not want to place anything on their skin, but elements with meaningful and actual value for themselves.
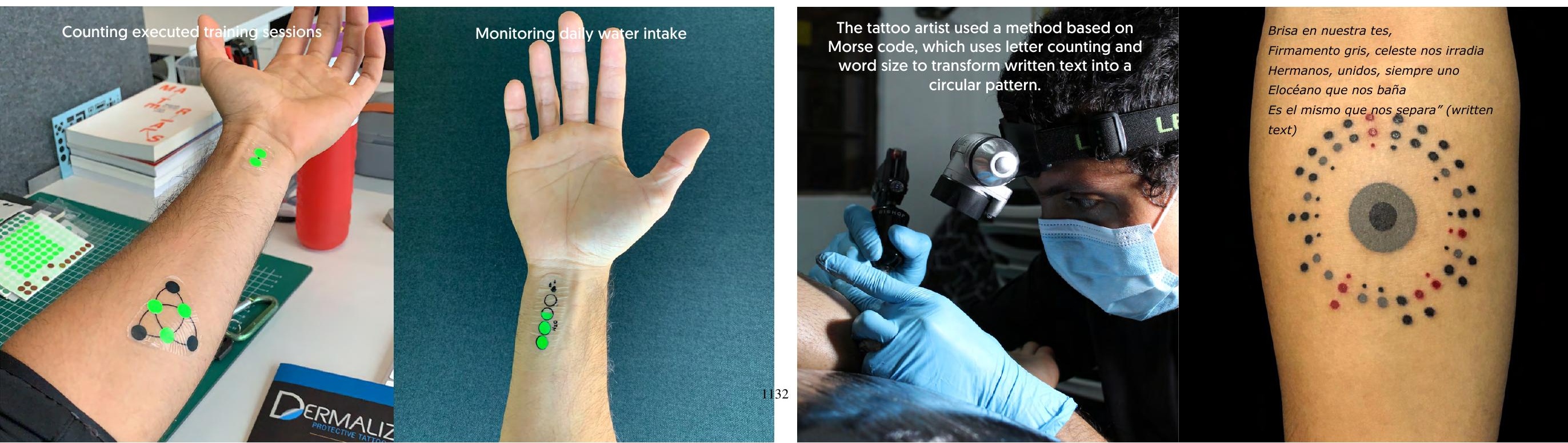


\section{INITIAL DESIGN PRINCIPLES}

The first-person explorations around personal data representation through OSI, tattooing and OSI applied to the context of wearables for running, gave us a holistic view of the elements composing the topic of OSI for sports. The fact that people tend to encrypt and not to make (fully) explicit the meaning of a tattoo is an important element to be considered into the design

of OSI. We propose that to succeed on developing a convincing, useful and appealing OSI, the gap between artificial and organic materiality and data representation must be bridged. To implement OSI in the context of recreational running, a balance between body location and accessibility for peripheral and direct interaction must be considered. Providing feedback on the part of the body that is impacted (e.g. knee impact resulting from the foot strike technique), as compared to an external non embodied interface, is an opportunity for design. According to this, we defined four initial design principles to frame our process and future exploration.

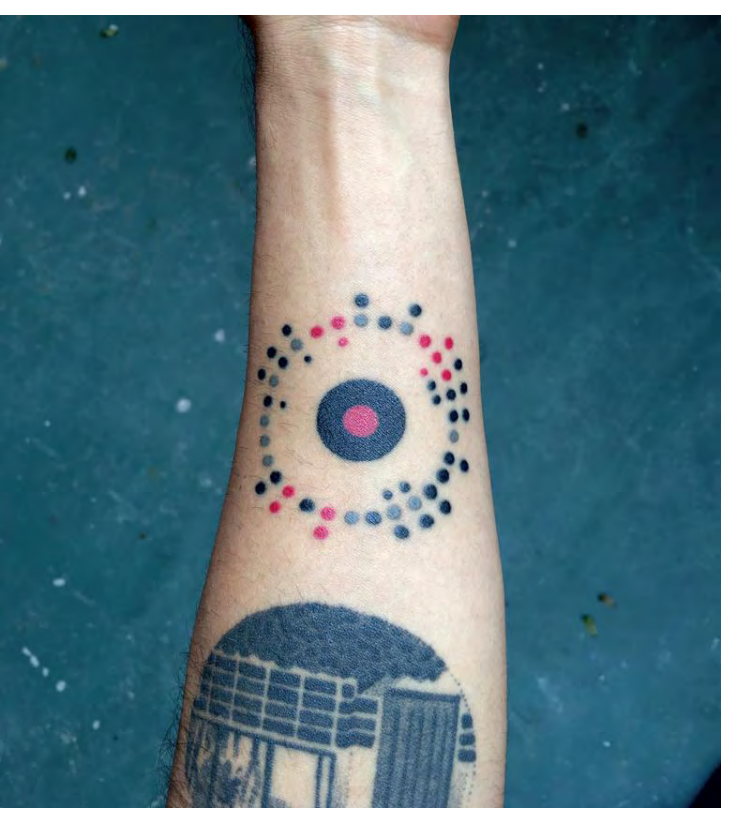

Meaningfulness and Privacy

The skin can be considered as a public interface. When designing OSI, access to the personal related information to the people in the vicinity must be restricted while being understandable and meaningful for the users themselves While open for interpretation, On-Skin Interfaces must keep their actual meaning private, only fully understandable for the user who "wears" it.

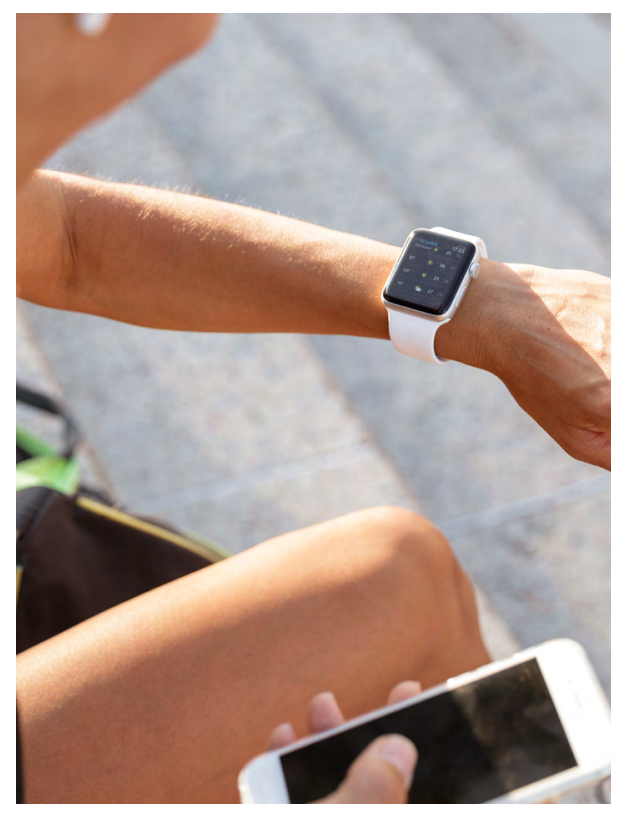

\section{Accessibility and Placement}

Alike "conventional" wearable devices, On-Skin Interfaces must be easy to understand and reach by the users. As the skin offers a myriad of body locations to install OSI for in-situ data representation, access and reachability must not be compromised

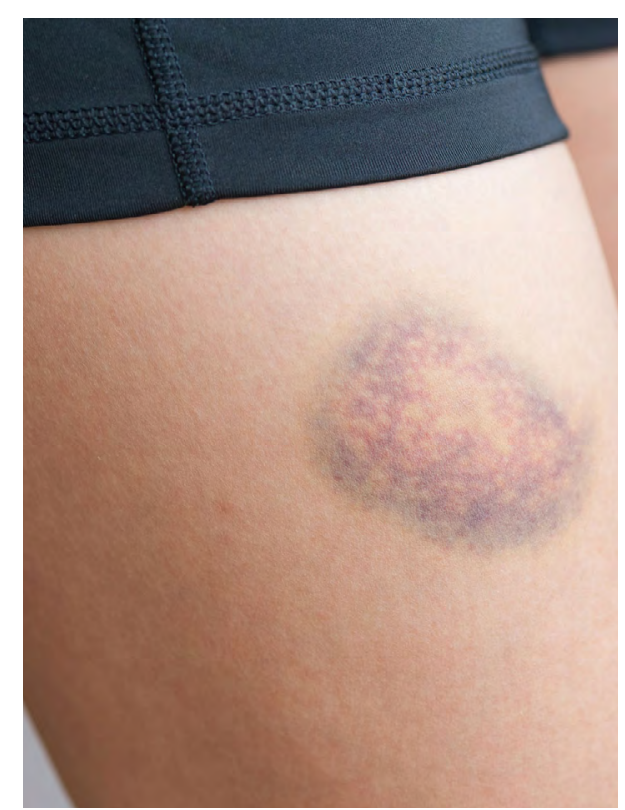

\section{Skin's Kinship}

Translating the properties of skin reactions such as skin bruises could enable On-Skin Interfaces to blend and be fully integrated within the human body. To be able to blend with the human body and to elicit an illusory feeling of body continuity, OSI must mimic the appearance, feeling, behavior and sensorial and physical properties of the human skin.

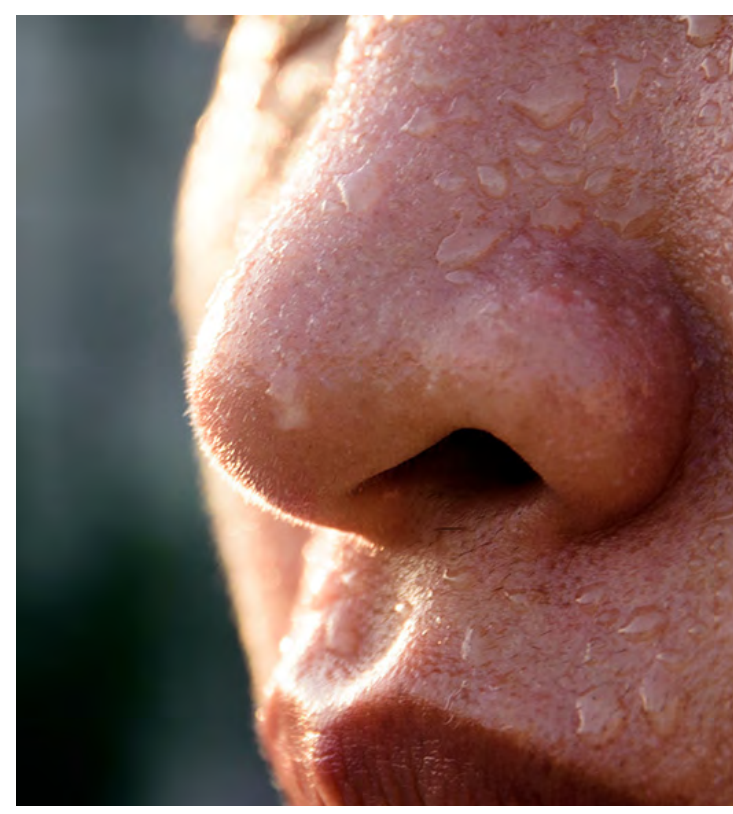

\section{Bodily relatedness}

Imagine a wearable interface using artificial sweating to provide feedback and bodily-related information to the users. This might open opportunities for richer and more intuitive interaction modalities. OSI must resemble and be inspired by the human body and its amazing sensing capabilities, responsive materials, and advanced organic mechanisms that offer intuitive, embodied and efficient feedback, experiences and interactions. 


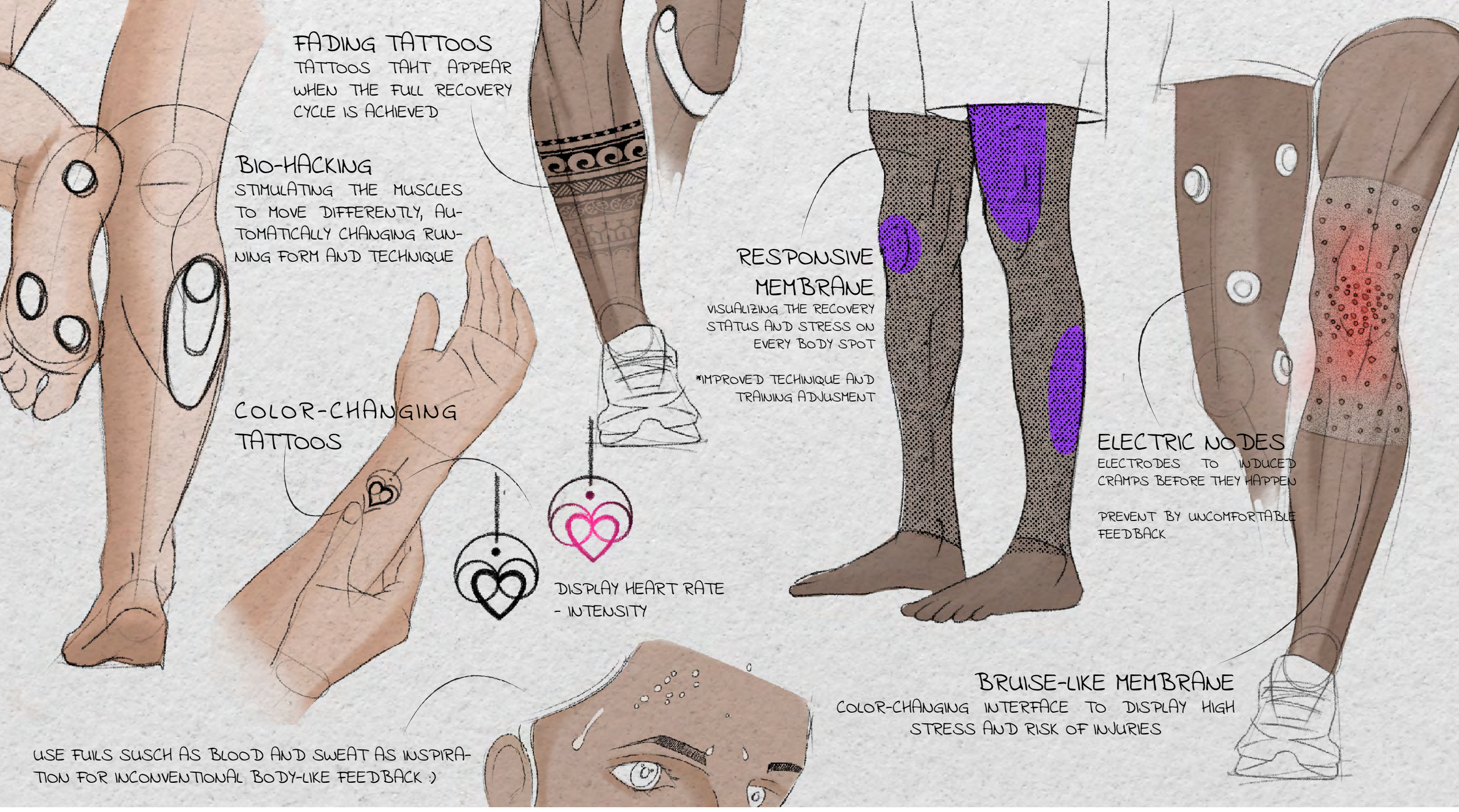

\section{SKETCHING DESIGN CONCEPTS}

Using our initial design principles, we ideated to design an On-Skin Interface able to represent biofeedback by mimicking bodily feedback mechanisms. For this, we sketched ideas that integrate elements of our four design principles within a single design. Sketching allowed us to overcome the technical and physical limitations of prototyping On-Skin Interfaces and to visualize a diverse set of design concepts within the context of recreational running. To keep the context of recreational running at the center of our exploration, the sketching exercise focused on sketching running human bodies, so the proposed concepts could be easily evaluated. This helped us to visualize possible shapes and relevant body locations for the OSI. Through the sketching explorations we aimed to define the best balance between form, feeling and location to represent bodily and runningrelated data directly on the skin of the users. 


\section{MATERIALITY EXPLORATION}

We used the insights from the sketching stage to inform the prototyping and materialization phase. For this, we defined two design concepts that compiled the most relevant aspects of the sketches.

\section{Color-Changing and Haptic Sensations}

The first concept was focused on using color changing and haptic sensation as two of the most recognizable skin-related feeling and feedback on external or internal bodily reactions and processes. We used a laser cut piece of EonTex Conductive fabric impregnated with thermochromic ink and encapsulated into two layers of transparent polyurathane and hypoallergenic acrylic glue. Due to the electric resistance of the EonTex, once electricity was applied to it, its fiber's temperature increased to reveal the shape of a heart hidden into the shape of the patch. The patch also produced a ticklinglike sensation on the skin, caused by the transference of micro-electric shocks through the adhesive film. The patch was controlled using heart-rate signal, as a basic and effective way to measure running intensity. While simple in terms of materiality and implementation, the combination of these materials was surprisingly effective, accessible and attractive to prototype quick but functional OSI. Both materials are versatile, durable to torsion and deformation. Similar to the findings of Pohl \& Hornbæk (2018) [28], the tickling sensation does a good job resembling skin and body's own dynamic feedback mechanisms. Yet, there are several limitations in terms of usability and safety. Sweat or water could either damage the components or alter their electrical properties. This could lead to major skin irritations and discomfort. It also requires a substantial power supply to work properly, limiting its use in the context of recreational running. The reaction time of the Eon Tex is rather slow and the thermochromic ink might be activated by external or internal factors such as weather or body temperature. This hinders the representation of real-time data, ideal to support runners on the go. Nevertheless, we produced meaningful insights for the next steps of our exploration and implemented by other researchers and designers interested in OSI. 


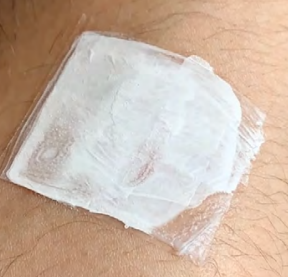

\section{Feeling and Visualizing Fluid-Related Reactions}

Inspired by the bodily fluid reactions such as sweat, blood and fluid swelling, we developed a patch able to use water-based liquids to display visual information and/or deliver a haptic sensation on the skin. We used layers of the same skin-friendly film used in the previous design and replaced the conductive membrane with absorbing, hydrochronic and screen-printed materials. The first prototype was a hydrochromic patch revealing a hidden pattern when in contact with a water-based fluid. The idea was to use the sweat coming out from the skin to activate the hydrochromic ink on the patch. The patch was tested during a long-distance indoor run by one of the researchers, who had experience with this type of exercise. To be as less intrusive as possible while easing visual access, the patch was placed on the forearm of the runner. The patch worked as expected, yet it took longer to get the patch fully wet, because the sweat was difficult to capture. This idea of using fluids nonetheless captured our interest as a very unconventional artificial feedback mechanism that is very common in nature. As the excess of sweat might be a cause of dehydration and high temperatures, this patch could be used during running events to remind runners to stay hydrated.

To overcome the challenges of capturing the sweat, we explored the idea of injecting liquid from an external source. We encapsulated absorbent materials such as thin felt and non-woven fibers into different skin-friendly sleeves. We injected a colored solution to the patches to test their absorbing, drying, and feeling properties. The sensation created by the liquid absorbed by the patch attached to the skin was very different compared to conventional haptics, such as pressure, touch, or vibration. As the dry patch was barely noticeable on the skin, the feeling created when the patch was getting wet was more like a bodily reaction than an external stimulus. The difference in temperature together with feeling the liquid flowing and making the patch heavier created a very organic and noticeable sensation. Interestingly, when the patch was full of liquid, it started to leak. Although this was an accident, the feeling of having liquid pouring out of the skin is alarming, uncomfortable, and could be compared to an external hemorrhage or wound. Yet, injecting liquid in the patch limits its interrupted use, increases its technical complexity, and hinders its seamless properties. This type of feedback could be implemented as an early warning mechanism, to alert runners of potential bodily damage or injury caused by, for instance, excessive effort or physical stress.

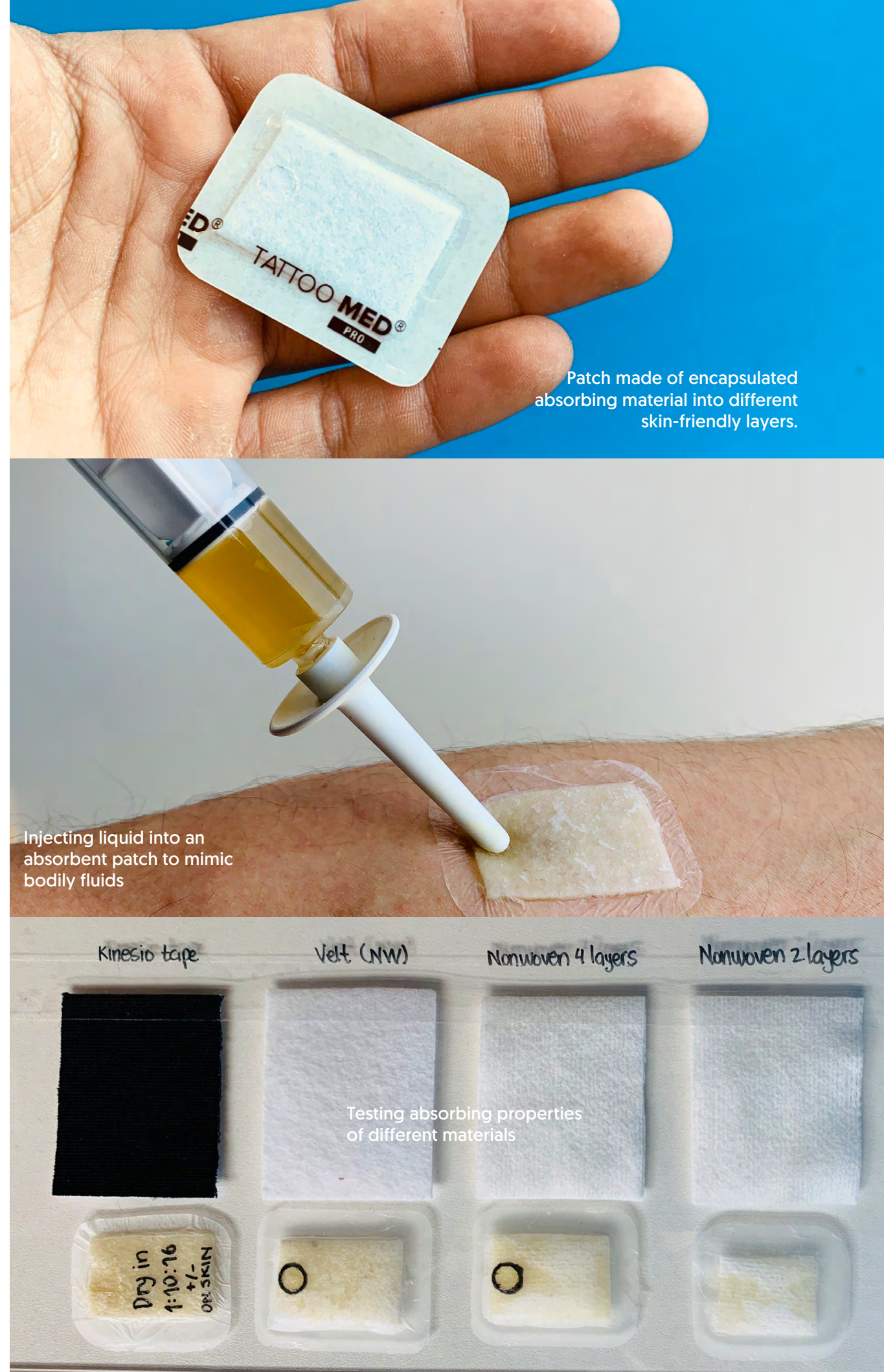




\section{ON-SKIN INTERFACES FOR RECREATIONAL RUNNING}

The previous prototyping activities informed our final OSI design. For this iteration, we merged the colorchanging and fluid properties from our materiality exploration into a one single OSI. We found in these two ways of materializing information, relevant and interesting elements to be implemented within the context of OSI for sports and recreational running. They also match the design requirements outlined by the four design principles formulated at the beginning of our prototyping journey. To create the OSI for recreational running, we created a hydrochromic and absorbing patch, able to deliver visual and haptic feedback. We screenprinted a geometric pattern on a piece of thin white felt and cover it with hydrochromic ink. We encapsulated all these components into a sleeve made of transparent polyutherane and hypoallergenic acrylic glue. To inject the water to the patch, we made a water pump, actuated by a servo motor controlled by an Arduino board. We

implemented the KneeIMPACT insole to measure foot-strike and activate the system. Two milliliters of water were injected into the patch when the insole measured continuous rear-foot strike for more than five consecutive minutes and continued injecting two milliliters every minute until the running technique was adjusted. The patch was designed to be attached close or next to the runner's knee in a vertical position. This to not interfere with the natural movement of the joint and to use gravity to control the flow direction of the water. The pattern printed used red dots that increased in size to convey higher impact. The dots were gradually revealed, from top to bottom, by the accumulation of water inside the absorbing material was enough to activate the hydrochromic layer covering the pattern.

Although we knew displaying visual cues on a runner's knee might not be efficient and perhaps

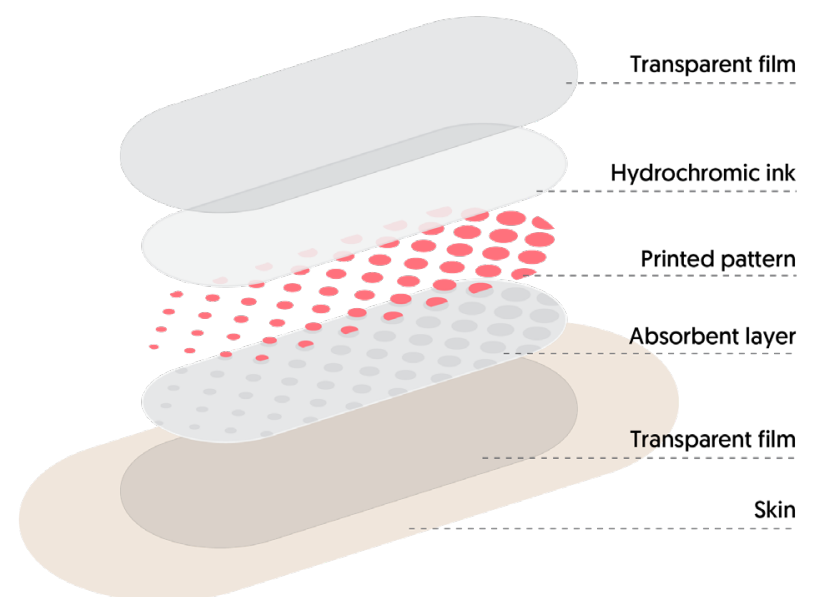

Skin

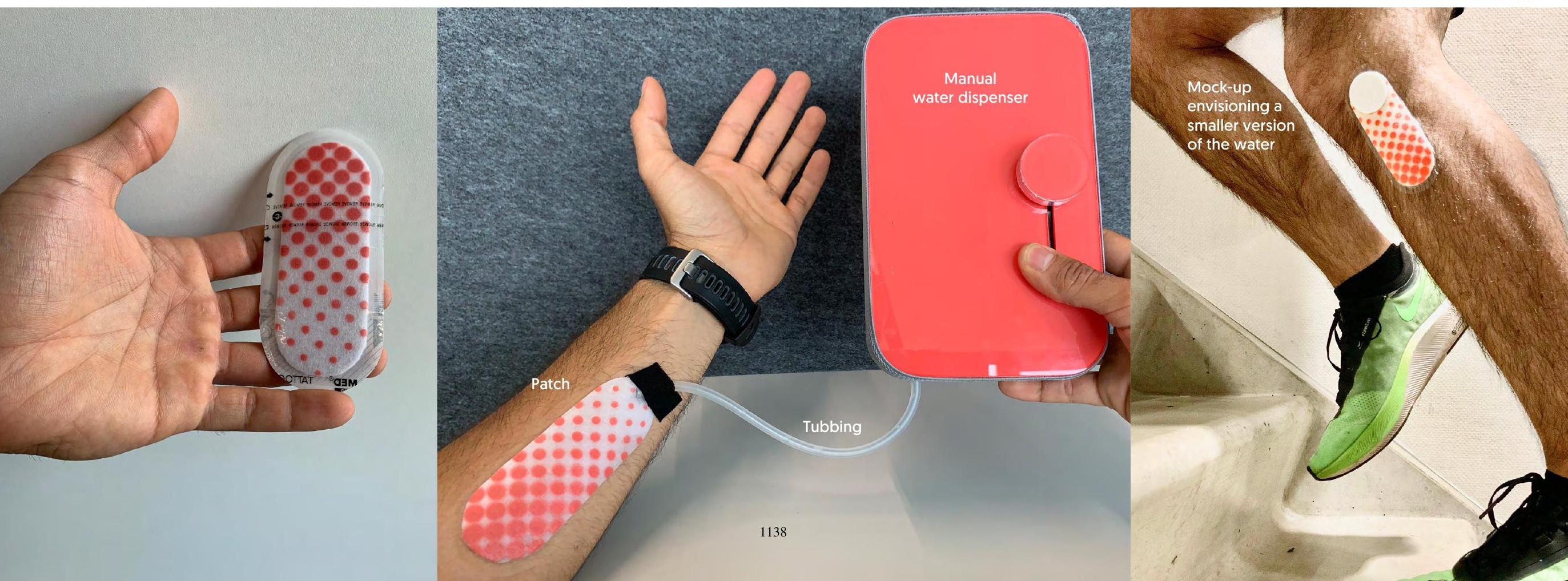


counterproductive, the aim of the dots was to reveal a pattern for the after running. This pattern could be used as an artificial bruise and early-warning mechanism, that combined with the watery feeling could inform the runner of the need of having proper rest and work on improving their running technique. Moreover, as the patch dries the pattern disapears, informing the user of the current status of the body and the interface itself.

\section{Running with OSI}

Once all the components of the system were working, we implemented them in the real context of use. One of the researchers wore the device during a full marathon event $(42.2 \mathrm{~km})$. As controlling the water pump was not safe, convenient and rather intrusive, we created a smaller version of the pump and connected the dispenser to the KneeIMPACT insole developed in the earlier stages of our prototyping journey. The KneeIMPACT implemented the same criteria previously described to measure foot-strike to activate the system. Thus, two milliliters of water were injected into the patch when the insole measured continuous rear-foot strike for more than five consecutive minutes and continued injecting two milliliters every minute until the running technique was adjusted. Besides the tube to inject the water into the patch and the extra weight of the water pump stored in the runner's hip pack, the patch was reported to be comfortable and not intrusive. Yet, the system was not triggered even once before the first 30 kilometers. This was most likely caused by the neutral technique of the runner. After the 35th kilometer, the system was trigger intermittently until the end of the race, probably due to the deterioration of the running technique caused by fatigue and exhaustion. The runner reported the patch to be clearly noticeable when it started to get wet and heavier, indicating the need of adjusting the running technique. The feeling produced by the patch was reported to be very similar to the feeling of sweating and getting blisters yet without the pain. The runner reported that the patch did a good job notifying the need of improving the running technique. Yet, by the last kilometers of the race, the runner did not feel the patch anymore. As it was a warm day, the water in the patch got both dry and acclimatized very quickly. Furthermore, the constant impact and vibration of the legs might have decreased the noticeability of the patch. Finally, the exhaustion and pain narrowed the focus of attention of the runner, where the patch was not a prioroty anymore but finishing the race.

During the running event, the patch exhibited the potential to be used by recreational runners, ideally running shorter distances. Although we used the system to monitor and represent foot-strike-related information, OSI are versatile devices that can easily be placed and accommodated on any spot of the skin and body shapes. Hence, OSI have the potential to be implemented in different sports scenarios and display any type of context-specific information. During our prototyping journey, we approached the design of OSI in a fully embodied fashion, learning directly from the practice and contact with the materials and body. During this process, we implemented four design principles to foreground our prototypes and explorations. While we do not propose these design principles as the ultimate principles for the design of OSI, we consider them relevant now of designing on-bodied systems aimed to represent self-related data.

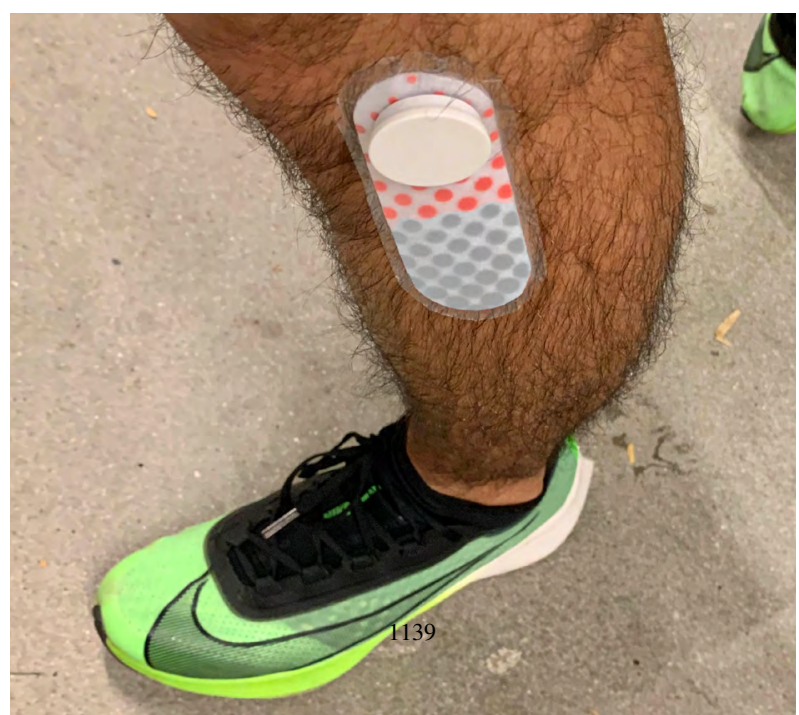

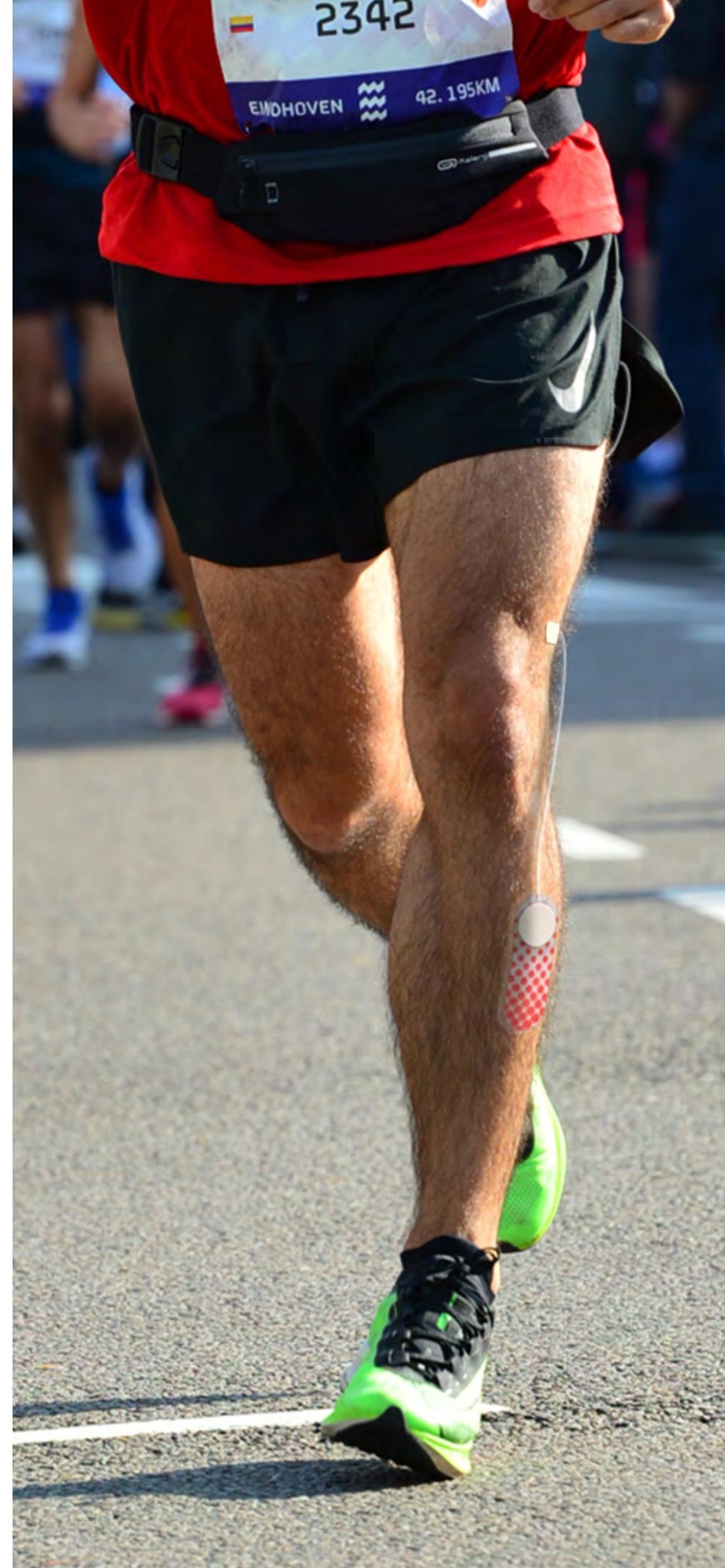




\section{REFLECTIONS}

\section{Studying wearability through first-person experiences}

In our design journey, we adopted a first-person perspective inspired by the work of Tomico et al., who call for a "[migration of] the design process from the technological oriented board to a full immersion into the design context". Using our own bodily experiences and senses helped us to gain insights on the challenging topic of OSI and to use their sensorial and material properties as a creative material for design. Exploring embodied practices and values related to the skin in a broader context than the one of recreational running was also insightful. For instance, working with a tattoo artist allowed us to better understand the factors that underline the skin modification and marking as a sublime and intimate process that goes beyond the appearance and digs into the aesthetics of interaction and semiotics.

\section{Design principles for OSI}

At the beginning of our journey, we defined four design principles to guide our design process. We now reflect and reframe them according to our experience, insights and related work in the field of OSI.

Body relatedness and skin's kinship: As these two principles define the overall purpose, materiality and execution of the OSI, we proposed to place them at a higher level within the design process. These represent the core values and differentiation factors between OSI and conventional wearable devices. We highlight the importance of focusing the design of OSI on the effort of resembling bodily-own processes and structures. For instance, Röddiger et al. [29] intend to mimic UI interface gestures on the skin. While this is a meaningful contribution, our intention is different. We aim to contribute to the augmentation of the human body by using its intrinsic properties and qualities. Through our explorations, we noticed that OSI inspired by organic and bodily principles had a higher probability to eventually blend within the body. As we strive to explore OSI and their potential association with a higher bodily self-consciousness [7], we need to design artefacts and interfaces that differentiate themselves from traditional devices. These can be achieved by facilitating experiences characterized by the immediate and continuous feeling that the body, its parts, and its sensations belong to oneself $[8,13,22]$. Röddiger et, al. [29] suggest that, by mimicking the haptic feeling of human skin on OSI devices these could almost be not perceivable by the user. We stand for a different perspective. We suggest that by achieving this, OSI devices will be perceived as own-body parts rather than external devices.

Regarding the Meaningfulness and Privacy and Accessibility and Location principles, we split them into three more specific principles, defined into usability and functionality principles for OSI as follows:

Meaningfulness: The skin not only protect us from the environment but also defines our identity and contact with the outside world. As we learned from the tattooing exercise and aligned with the work of You et al. [42] and Withana et al. [40], the design of OSI must facilitate the customization and representation of meaningful on-body information according to the cultural values, social factor and personal taste of individuals.

Privacy: Considering the skin as a public interface has several design implications. As we experienced, it is common for people to hide and encrypt meaning in their tattoos. As tattoos often represent personal experiences or intimate information, they are rarely literal representations of the meaning they contain. We propose to approach the privacy component of OSI in the same fashion. As suggested by You et al. [42], the social and public perception of using OSI potentially determines the user acceptance of this technology. The implications of OSI in terms of privacy should of course be considered according to their context of implementation [28, 42]. The cultural and social dynamics might influence their acceptance, interpretation and use.

Location (/accessibility): As the skin offers a wide range of body locations where the OSI can be placed, using feedback and representation modalities that rely on more than one sense is crucial from successful OSI. As we learned from our running-related activities, the access to the feedback and information delivered by OSI systems must be fluent, clear and consistent. Furthermore, and alike the privacy, the location might be influenced by cultural, social and personal factors that must be considered before starting the design of OSI. Some people would prefer to place OSI in body spots that are not cover by clothing, yet this differs across cultural backgrounds [23, 42]. In this sense, a balance between usability properties and contextual factors is essential.

\section{AKNOWLEDGEMENTS}

This research is part of Citius Altius Sanius, a project supported by the Dutch Organization for Scientific Research (NWO) to encourage injury-free exercise for everyone. 


\section{REFERENCES}

1. Amay J. Bandodkar, Wenzhao Jia, Ceren Yardımcı, Xuan Wang, Julian Ramirez, and Joseph Wang. 2014. Tattoo-based noninvasive glucose monitoring: a proof-of-concept study. Analytical chemistry, 87(1), 394-398.

2. Joanna Bergstrom-Lehtovirta, David Coyle, Jarrod Knibbe, Kasper Hornbæk. 2018. I Really did That: Sense of Agency with Touchpad, Keyboard, and On-skin Interaction. In Proceedings of the 2018 CHI Conference on Human Factors in Computing Systems (pp. 1-8).

3. Joanna Bergstrom-Lehtovirta, Sebastian Boring, Kasper Hornbæk. 2017. Placing and recalling virtual items on the skin. In Proceedings of the 2017 CHI Conference on Human Factors in Computing Systems (pp. 1497-1507).

4. Heidi R. Biggs \& Audrey Desjardins. 2020. Crafting an Embodied Speculation: An Account of Prototyping Methods. In Proceedings of the 2020 ACM Designing Interactive Systems Conference (pp. 547-560).

5. Adam I. Daoud, Gary J. Geissler, Frank Wang, Jason Saretsky, Yahya A. Daoud, And Daniel E. Lieberman (2012). Foot strike and injury rates in endurance runners: a retrospective study. Med Sci Sports Exerc, 44(7), 1325-1334.

6. Jordan Eschler, Arpita Bhattacharya, Wanda Pratt. 2018. Designing a reclamation of body and health: cancer survivor tattoos as coping ritual. In Proceedings of the 2018 CHI Conference on Human Factors in Computing Systems (pp. 1-12).

7. Tieri Gaetano, Emmanuele Tidoni, Enea Francesco Pavone, Salvatore Maria Aglioti. 2015. Body visual discontinuity affects feeling of ownership and skin conductance responses. Scientific reports, 5, 17139.

8. Shaun Gallagher. 2000. Philosophical conceptions of the self: implications for cognitive science. Trends in Cognitive Sciences, 4(1), 14-21.
9. Marlène Giandolini, Pierrick J. Arnal, Guillaume Y. Millet, Nicolas Peyrot, Pierre Samozino, Blaise Dubois \& Jean-Benoît Morin (2013). Impact reduction during running: efficiency of simple acute interventions in recreational runners. European journal of applied physiology, 113(3), 599-609.

10. Mahmoud Hassan, Florian Daiber, Frederik Wiehr, Felix Kosmalla, F, Antonio Krüger. 2017. Footstriker: An EMS-based foot strike assistant for running. Proceedings of the ACM on Interactive, Mobile, Wearable and Ubiquitous Technologies, $1(1), 2$.

11. Kristina Höök, Baptiste Caramiaux, Cumhur Erkut, Jodi Forlizzi, Nassrin Hajinejad, Michael Haller, Caroline Hummels, Katherine Isbister, Martin Jonsson, George Khut, Lian Loke, Danielle Lottridge, Patrizia Marti, Edward Melcer, Florian Müller, Marianne Petersen, Thecla Schiphorst, Elena Segura, Anna Ståhl, Dag Svanæs, Jakob Tholander, and Helena Tobiasson. 2018. Embracing First-Person Perspectives in Soma-Based Design. Informatics 5, 1: 8. https://doi.org/10.3390/ informatics 5010008 .

12. Kristina Höök. 2018. Designing with the Body: Somaesthetic Interaction Design. MIT Press.

13. Behavioural Brain Research 2003;142(1-2):115.

14. Frances Joseph, Miranda Smitheram, Donna Cleveland, Caroline Stephen, and Hollee Fisher. 2017. Digital materiality, embodied practices and fashionable interactions in the design of soft wearable technologies. International Journal of Design 11, 3: 7-15.

15. Frances Joseph. 2017. Redressing perspectives: Mediation, embodiment, and materiality in digital fashion and smart textiles. Necsus: 1-36.

16. Joohee Jun, Myeongul Jung, So-Yeon Kim, Kwanguk(Kenny) Kim. 2018. Full-Body Ownership Illusion Can Change Our Emotion. In Proceedings of the 2018 CHI Conference on Human Factors in Computing Systems (p. 601). ACM.

17. Hsin-Liu (Cindy) Kao, Abdelkareem Bedri, Kent Lyons. (2018). SkinWire: Fabricating a SelfContained On-Skin PCB for the Hand. Proceedings of the ACM on Interactive, Mobile, Wearable and Ubiquitous Technologies, 2(3), 1-23.

18. Hsin-Liu (Cindy) Kao, Christian Holz, Andres Calvo, Chris Schmandt. 2016. DuoSkin: rapidly prototyping on-skin user interfaces using skinfriendly materials. In Proceedings of the 2016 ACM International Symposium on Wearable Computers (pp. 16-23). ACM.

19. Henry Lin, Ron Wakkary, and Doenja Oogjes. 2019. The Tilting Bowl: Electronic design for a research product. DIS 2019 - Proceedings of the 2019 ACM Designing Interactive Systems Conference (pp. 345-357).

20. Xin Liu, Katia Vega , Pattie Maes, Joe A. Paradiso (2016, February). Wearability factors for skin interfaces. In Proceedings of the 7th Augmented Human International Conference 2016 (pp. 1-8).

21. Lian Loke and Toni Robertson. 2010. Studies of Dancers: Moving from Experience to Interaction Design. International Journal of Design 4, 1934.

22. Christopher Lopez, Pär Halje, and Olaf Blanke. 2008. Body ownership and embodiment: vestibular and multisensory mechanisms. Neurophysiologie Clinique/Clinical Neurophysiology, 38(3), 149-161.

23. Lara Maistera, Natalie Sebanzbc, Günther Knoblichb, ManosTsakirisa. 2013. Experiencing ownership over a dark-skinned body reduces implicit racial bias. Cognition, 128(2), 170-178.

24. Helena Mentis, Katherine Isbister, Kristina Höök, George Poonkhin Khut, Florian Mueller, and Toni Robertson. 2014. Designing for the experiential body. In Proceedings of the 2014 Conference on Human Factors in Computing Systems, 1069-1073. 
https://doi.org/10.1145/2559206.2579402.

25. Florian 'Floyd' Mueller, Richard Byrne, Josh Andres, Rakesh Patibanda. 2018. Experiencing the body as play. In Proceedings of the 2018 CHI Conference on Human Factors in Computing Systems (pp. 1-13).

26. Carman Neustaedter and Phoebe Sengers. 2012. Autobiographical Design in HCI Research: Designing and Learning through Use-It-Yourself. Proceedings of the 2012 Designing Interactive.

27. William Odom, Ishac Bertran, Garnet Hertz, Henry Lin, Amy Yo Sue Chen, Matt Harkness, and Ron Wakkary. 2019. Unpacking the thinking and making behind a slow technology research product with slow game. Proceedings of the 2019 Creativity and Cognition (C \& C '19): 15.

28. Henning Pohl, Kasper Hornbæk. 2018. October. Electricitch: Skin irritation as a feedback modality. In Proceedings of the 31st Annual ACM Symposium on User Interface Software and Technology (pp. 765-778).

29. Tobias Röddiger, Michael Beigl, Daniel Wolffram, Matthias Budde, Hongye Sun. 2020. PDMSkin: On-Skin Gestures with Printable Ultra-Stretchable Soft Electronic Second Skin. In Proceedings of the Augmented Humans International Conference (pp. 1-9)

30. Paula Roinesalo, Tuomas Lappalainen, Ashley Colley, and Jonna Häkkilä. 2017. Breaking of the dawn jacket. Proceedings of the 2017 ACM International Symposium on Wearable Computers - ISWC '17: 232-237. https://doi. org/10.1145/3123021.3123074.

31. Robert Rosenberger, Peter P.C.C. Verbeek. 2015. Postphenomenological investigations: essays on human-technology relations. Lexington Books.

32. Anna Ståhl, Martin Jonsson, Johanna Mercurio, Anna Karlsson, Kristina Höök, and Eva Carin
Banka Johnson. 2016. The soma mat and breathing light. Conference on Human Factors in Computing Systems - Proceedings 07-12-May-: 305-308. https://doi.org/10.1145/2851581.2889464.

33. Jürgen Steimle. (2016). Skin--The Next User Interface. Computer, 49(4), 83-87.

34. Jürgen Steimle, Joanna Bergstrom-Lehtovirta, Martin Weigel, Aditya Shekhar Nittala, Sebastian Boring, Alex Olwal, Kasper Hornbæk. 2017. On-skin interaction using body landmarks. Computer, 50(10), 19-27.

35. Oscar Tomico and Danielle Wilde. 2016. Soft, embodied, situated \& connected: enriching interactions with soft wearables. mUX: The Journal of Mobile User Experience: 1-17. https://doi. org/10.1186/s13678-016 0006-z.

36. Vasiliki Tsaknaki, Madeline Balaam, Anna Ståhl, Pedro Sanches, Charles Windlin, Pavel Karpashevich, and Kristina Höök. 2019. Teaching Soma Design. 1237-1249. https://doi. org/10.1145/3322276.3322327.

37. Stephan Wensveen, Tom J.P. Djajadiningrat, Kees C.J. Overbeeke. 2004. Interaction frogger: a design framework to couple action and function through feedback and feedforward. In Proceedings of the 5th conference on Designing interactive systems: processes, practices, methods, and techniques (pp. 177-184). ACM.

38. Danielle Wilde, Thecla Schiphorst, and Sietske Klooster. 2011. Move to design/design to move. Interactions $18,4: 22$. https://doi. org/10.1145/1978822.1978828.

39. Dejan Wilde. 2008. Gestalt principles. Scholarpedia, 3(12), 5345 .

40. Anusha Withana, Daniel Groeger, Jürgen Steimle. 2018. Tacttoo: A thin and feel-through tattoo for on-skin tactile output. In The 31st Annual ACM Symposium on User Interface Software and
Technology (pp. 365 378). ACM.

41. Anusha Withana, Daniel Groeger, Jürgen Steimle. 2018. Tacttoo: A thin and feel-through tattoo for on-skin tactile output. In Proceedings of the 31 st Annual ACM Symposium on User Interface Software and Technology (pp. 365-378).

42. Chuang-Wen You, Ya-Fang Lin, Elle Luo, Hung-Yeh Lin, Hsin-Liu (Cindy) Kao. 2019. Understanding social perceptions towards interacting with on-skin interfaces in public. In Proceedings of the 23rd International Symposium on Wearable Computers (pp. 244-253). 